\title{
OLFACTORY SENSE AS AN \\ OBJECT OF DESIGN \\ PRACTICE:DESIGNING FOR AN \\ EMOTIONAL EXPERIENCE IN \\ THE SMART TECHNOLOGY \\ SECTOR
}

Cecilia Lee

Royal College of Art / Kensington Gore, London SW7 2EU, United Kingdom cecilia.lee@network.rca.ac.uk 


\section{Overview}

Despite the mainstreaming of smart devices, the user abandonment remains an unresolved challenge. It is easy to come across users who have stopped using their smart devices, whether it be a smart watch or a voice assistant. In this study, it explores the olfactory sense as an object of design practice to examine how designers can use the olfactory sense to design for an emotional experience between a user and a smart device. The study takes a research through design approach and uses a design experiment as a research method. The aim of the study is to investigate how olfactory stimulus and its interaction effect with visual stimulus influence the user's emotional response during the interaction with an Al chatbot and the user's willingness to continue to use an Al chatbot in the future.

\section{Keywords}

User abandonment; smart device; olfactory sense; emotions; and design experiment

\section{Main Report}

\section{Research Problem and Motivation}

The recent waves of smart technologies such as artificial intelligence (AI) and the Internet of Things (IOT) have rapidly shifted a service economy towards a smart service economy. In smart service economy, not only can technologies autonomously interact with users, but also with other technologies. The material agency (Leonardi ,2011) granted to these technologies continuously refines their level of intelligence. Despite the devices becoming smarter each day, many of them still lack the ability to engage with users in a long run. The lack of user engagement in smart technology sector is already a well-reported case, especially in consumer wearables sector. Gartner's research report in 2016 claims that 30\% of fitness band owners abandon their devices after a few months of use.

Smart devices are often understood as the types of devices that can autonomously interact with users based on contextual information. The notable examples are fitness bands, smart watches and voice assistants. This study conducted initial interviews with ten individuals who own smart devices and no longer use them as frequently as before or have abandoned them entirely. The interviewees have pointed out a number of different reasons that have prevented them from continuously engaging with their devices, namely the lack of meaningful data, expectation mismatch, and the lack of personalisation. The findings from these interviews are also supported by previous studies that have examined why users often fail to engage with a smart device in a long run (Clawson et al, 2015; Lazar et al, 2015).

These reasons can be summarised as the lack of user's perceived value in using a device on a regular basis. Also, many interviewees have described the lack of value, not only in a utilitarian term but also in an emotional term, as some of them have explicitly described their interaction with their device as the absence of a 'human touch'.

Despite such observations, the body of extant literature has yet to have examined how to design for an emotional experience between a user and a smart device. The majority of past studies have focused on designing for an experience that prompts utilitarian value creation between a user and a technology (Lenz et al, 2014). In order for designers to design for an 
emotionally engaging experience between a user and a smart device, they should focus on the meanings emerged through the interaction between these two actors rather than the use of technology only.

Previous studies have demonstrated that users who have experienced positive emotions are more likely to evaluate their experiences positively (Bolton et al, 2014; Zomerdijik and Voss, 2010) which often results in a loyalty behaviour. Designers can play an important role here by creating experiential touchpoints that can modulate an emotional response in users during the user's interaction with a smart device.

The human's olfactory sense is known to have a direct link to the limbic system of the human brain that controls the human emotions (Obris et al, 2017). Despite its powerful role as a stimulus to trigger an emotional response in human, olfactory sense has been significantly under-investigated in the extant literature.

Therefore, this study examines the olfactory sense as an object of design practice and explores how designers can use the olfactory stimulus to design for an emotionally engaging experience between a user and a smart device.

\section{Relevant Past Studies}

Past studies in human-computer interaction $(\mathrm{HCl})$ that look at the role of human senses in experience have focused mostly on visual, auditory and haptic senses (Desmet and Hekkert, 2007). Although $\mathrm{HCl}$ researchers have examined olfactory sense since a decade ago, these studies are only interested in investigating whether olfactory stimuli can serve the functional purpose of a device more effectively, such as an olfactory-based medium of communication (Xiang et al, 2016) or a notification (Bodnar et al, 2004). As a result, the current knowledge still lacks insights on how the olfactory sense can be used as an object of design for emotional experience design.

In response to this gap, this study aims to explore the following research question:

RQ: How can designers use the olfactory sense as an object of design practice to design for an emotional experience between a user and a smart device?

This research question is explored through two hypotheses below which were generated through the literature review. The body of extant literature argues that those who have experienced positive emotions are likely to evaluate their experiences positively (McCollKennedy et al, 2017; Bolton et al, 2014; Zomerdijik and Voss, 2010).

In line of this argument, this study has developed the first hypothesis is as follows:

$\mathrm{H1}$ : Olfactory stimulus triggers positive emotions in users frequently during the interaction with an Al chatbot which leads to an enhanced level of the user engagement.

Building on the first hypothesis, the second hypothesis below aims to validate whether the olfactory stimulus can prompt the value co-creation process between a user and an $\mathrm{Al}$ 
chatbot which often results in a positive future behavioural intention. Therefore, the second hypothesis is as follows:

$\mathrm{H} 2$ : Olfactory stimulus positively influences the user's value co-creation process with an $\mathrm{Al}$ chatbot that often leads to a positive future behavioural intention.

In order to test these hypotheses, this study has built an Al chatbot which can be used as a 'chatbot for emotional regulation'. Many researchers working in academia as well as the Millennnials are known to suffer from mental health issues such as depression and anxiety (Bira et al, 2019; Landrum, 2017). There are a number of chatbots (e.g. Woebot, Replika) that were rolled out to tackle this problem, but many of them are still not able to hold a natural conversation with a user. In this study, it has taken a Wizard of $\mathrm{OZ}{ }^{1}$ approach to enable the experimenter to control the back-end of the bot from other room, while each participant is exposed only to the front-end of the bot in the lab.

\section{Methodology}

This study takes a research through design approach (Frayling, 1993) which uses design experimentation as a method in this study context.

Research through design is a generative design research approach which uses design practices as a research method. It aims to explore various possibilities for the problem under study and ultimately look for the solution that will make the current situation into the preferred state of the future (Koskinen et al, 2011). During the pilot experiment, the data have been gathered through three different methods - a self-reported questionnaire, an emotional mapping exercise, and an exit interview - at three different phases of the user journey as illustrated below.

Figure 1: User Journey Map

\begin{tabular}{c|c|c} 
Pre-Engagement & During-Engagement & Post-Engagement \\
\hline A Self-Reported Questionnaire & Emotional Mapping & $\begin{array}{c}\text { A Self-Reported Questionnaire } \\
\text { Exit Interview }\end{array}$
\end{tabular}

A self-reported questionnaire was developed based on the Theory of Consumption Value (Sheth et al, 1991) which assesses three dimensions of user value - utilitarian value, emotional value, and a future behavioural intention. Each question was developed based on a 5-point Likert scale in which 5 represents strongly agree and 1 strongly disagree. The emotional map that was used for a mapping exercise was developed based on Russell's (1980) Circumplex Model of Affection which measures emotions based on two axes - the level of valence and of the arousal. At the end of chat session with an Al chatbot, each participant was invited to a 15 minutes of an exist interview in a semi-structured format which gave him and her a chance to think out loud and articulate their emotional map. All

\footnotetext{
${ }^{1}$ A Wizard of OZ approach is a term coined by J.F. Kelley in 1980. It is often used in the experimental psychology, human factors, ergonomics, and usability engineering disciplines to describe a testing or iterative design methodology in which an experimenter (the Wizard) simulates the behaviour of a theoretical intelligent computer application (Kelley, 1984).
} 
the interviews were transcribed and coded. The coded data were further sorted through the affinity diagram.

They were also asked to complete the same questionnaire that they had completed during the pre-engagement stage. In doing so, this study aims to uncover if there were any changes in user's perceived value of using an Al chatbot before and after their direct interaction with an Al chatbot.

\section{Procedure}

The Call for Participant was circulated across the Royal College of Art and Imperial College London, and it recruited ten students that consist of three males and seven females. The participant criteria for this study included the age group of the Millennials (18-35) who are postgraduate students and have experienced mild depression in the past three months.

Prior to running this pilot experiment, the pre-pilot test was conducted at the Royal College of Art with 22 students. The students are those who have responded to the Call for Scent Testing, and they sampled ten different odours and answered a short questionnaire that asks questions about the level of pleasantness, intensity, and familiarity of each odour. Each participant also shared any feelings or thoughts that had struck their mind while they were sampling each odour.

The chatbots have been developed in three different versions with a varying degree of visual stimulus. Given that the beauty is a highly subjective term, this study has developed chatbots in three different versions to allow participants to choose the bot they find attractive and would like to chat with. By doing so, this study has controlled the effect of visual stimulus on how each participant evaluates an interactive experience.

Figure 2: Al Chatbots

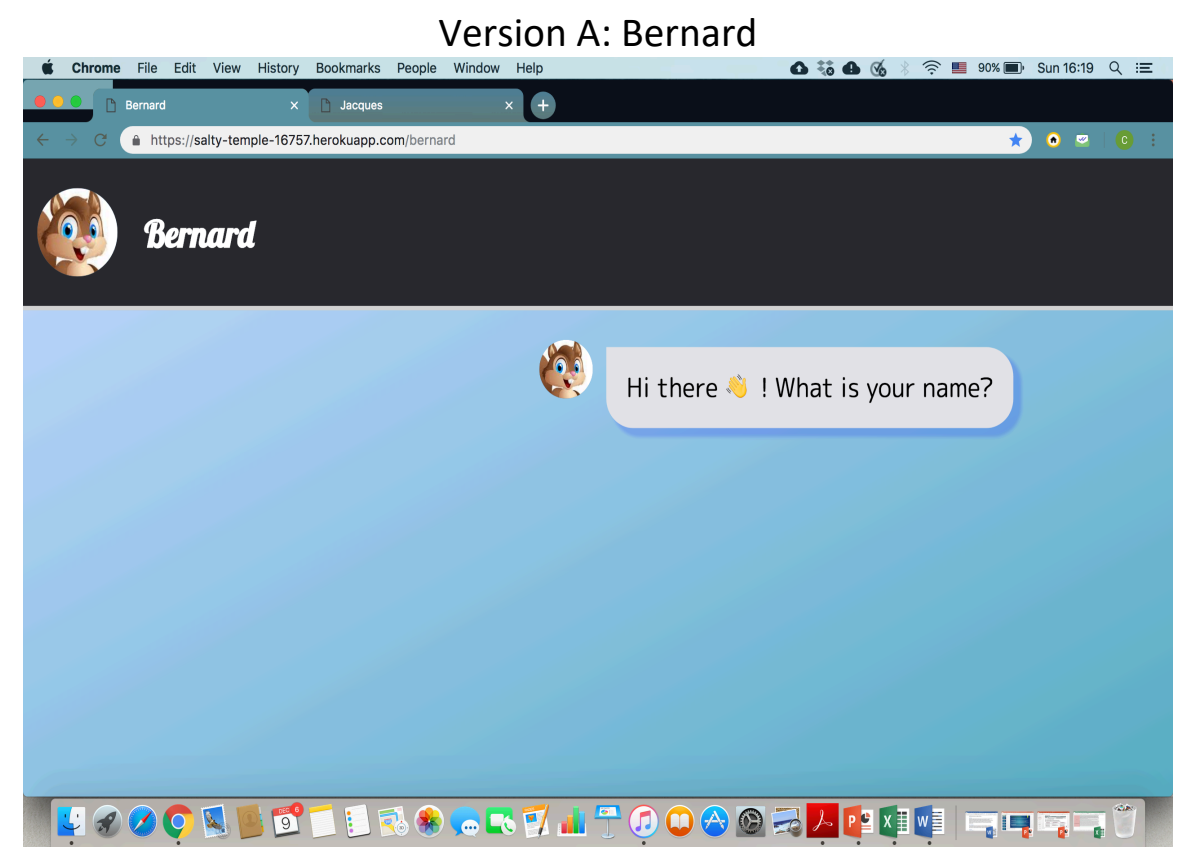


Version B: Jacques

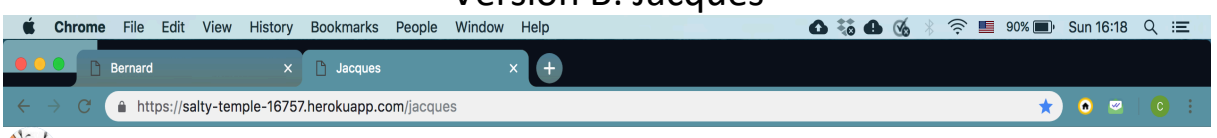

\section{Jacques}

Hi there ! What is your

name?

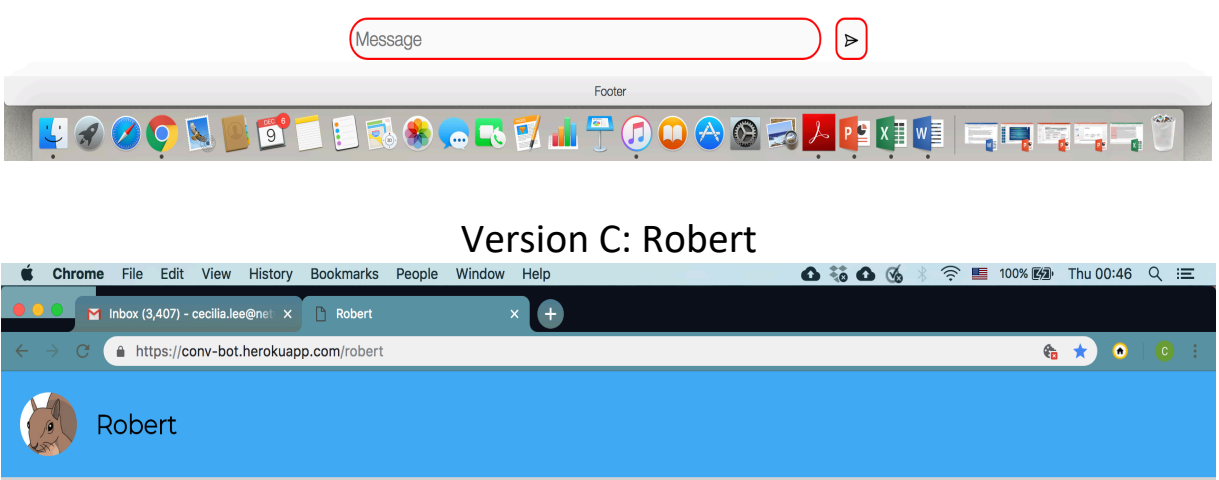

Hi there ! What is your name?

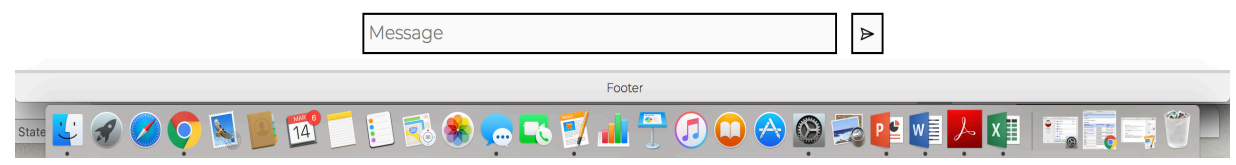

Of ten participants, five were randomly allocated to the control group without the olfactory stimulus, while the other five participants were allocated to the treatment group with the olfactory stimulus. For the treatment group, an aroma diffuser was installed into the room, and the diffuser was switched on an hour prior to the experiment. The pre-pilot test confirms that the majority of the participants found a vanilla scent as the most pleasant one. As a result, a vanilla scent was used as the olfactory stimulus for the pilot experiment.

First, each participant was asked to read and sign the consent form and answer a selfreported questionnaire that assesses each participant's perceived effect of visual and 
olfactory stimulus on their interaction with a chatbot. The participants were then asked to choose the chatbot they would like to chat with.

Before starting a chat session with a bot, they were asked to draw their current emotional status on Russell's model (1980). Starting from the current emotional status on the emotional map, they were asked to continue drawing their emotional change during the interaction with a bot . All participants chatted with a bot for 15 minutes. The conversation is loosely structured around each participant's school life in general, what kind of concerns they have for now, and what types of support might help them to stay psychologically healthy.

Upon the completion of the chat, the participants were asked to complete the same selfreported questionnaire they had completed during the pre-engagement stage. Finally a 15minute exit interview was conducted. This interview was held for the purpose of giving participants a chance to think out loud how their emotional response has evolved over the pre-, during-, and post-engagement stages.

\section{Results}

Of the total ten maps, the map 1 and 10 were excluded from data analysis, as they were not usable due to technical errors experienced during the experiment.

\section{A Self-Reported Questionnaire}

The data reveal that the user satisfaction in the treatment group has improved in the postengagement phase, so has the user's willingness to use the bot again in the future. In contrast, the user satisfaction in the control-group has not changed in the post-engagement phase, while the user's willingness to use the bot again in the future has diminished in the post-engagement stage.

\section{Emotional Maps}

Figure 3: Emotional Mapping

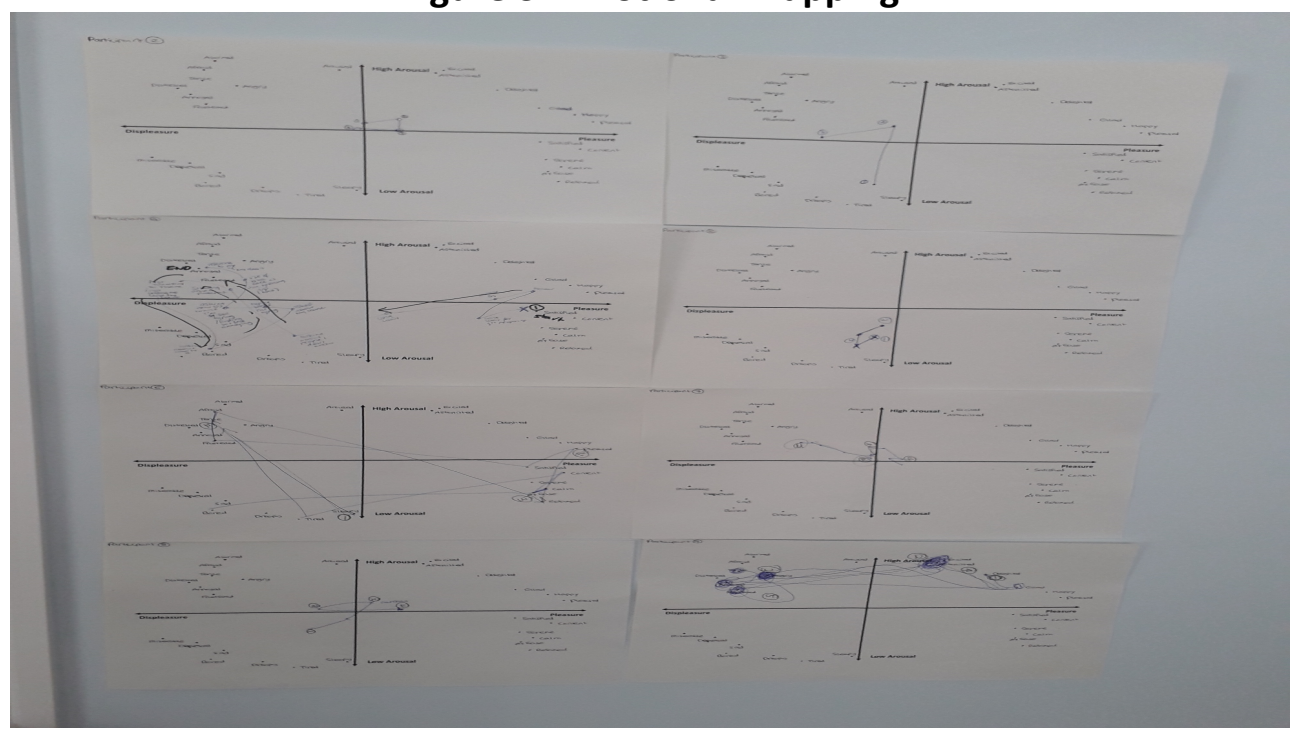

The Users' Emotional Maps with Russell's Circumplex Model (1980) 


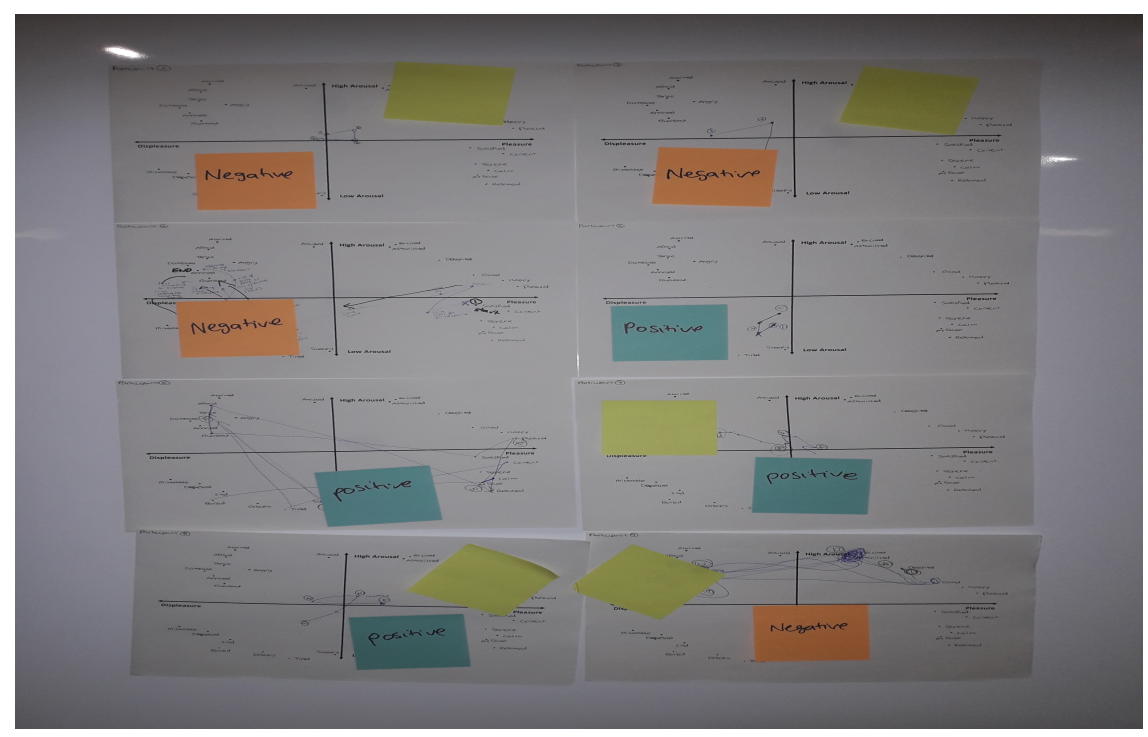

The Emotional Maps from the Treatment Group with Yellow Sticky Notes.

The data suggest that the treatment group participants' emotional responses are tilted towards the positive quadrants of the maps, as the chat session with the bot is unfolded. But most users in the treatment group finished the session with negative emotions. In contrast, the control group users finished the session with positive emotions, but their maps are rather skewed towards the negative quadrants.

\section{Exit Interview}

During the interview, the participants who finished the session with negative emotions confessed that they wanted to chat with a bot longer. When the bot said that the time is up after 15 minutes, two participants explained that they were sad, while another participant described his experience as annoying.

The tables 1 and 2 below showcase the five key themes emerged in the datasets from exist interviews. The key themes are sorted into the engaging and disengaging categories. The table 3 presents emotions triggered by olfactory and visual stimulus which are also sorted and grouped into the engaging and disengaging categories.

Table 1: Engaging Category 


\section{Discussion}

Several findings in the tables above depict a conflicting view. For example, some participants confessed that they can trust an Al bot more than a human therapist who tends to influence patients with their own personality, while some others claimed the opposite, saying that they felt that the bot was asking pre-determined questions. Since the experimenter directly controlled the back-end of the bot, the participants' responses were clearly influenced by their preconception of 'chatting with a bot, not a human.'

Some observations on emotions triggered by visual and olfactory stimulus and their effect on user experience suggest that visual stimulus of the bot strongly influenced the user's perceived value of using an Al bot only prior to the user's interaction with a bot, because its effect has quickly dissipated, as the user started chatting with the bot. The effect of olfactory stimulus discreetly influenced the user's emotions in a positive way. Although most participants claimed that olfactory stimulus did not influence their emotions during their interaction with a bot, their emotional maps suggest that their emotions were influenced by the intervention of the olfactory stimulus.

\section{Contributions}

This study makes contributions in three ways. First, the study delves into the olfactory sense which is undermined as a research topic. The findings from the pilot experiment can serve as preliminary insights for designers who would like to use the olfactory sense as an object of design practice in their design process. Second, it is an original contribution which provides guidance on emotional experience design for human-computer interaction. Lastly, the study takes a research through design approach to illustrate how a design experiment makes a legitimate method for exploratory research.

\section{Acknowledgment}

This study is supervised by Dr. Ronald Jones and Dr. Nick Leon at the Royal College of Art in London, UK. Prof. Barry Smith and Harry Sherwood from the University of London have provided support for the execution of the scent experimentation.

\section{References}

Amores, Judith and Maes, Pattie. 2017. "Essence: Olfactory Interfaces for Unconscious Influence of Mood and Cognitive Performance. CHI 2017, Denver, CO, USA. 6-11 May, 2017. 
Bell, Clive. (1914). Art. London: Chatto \& Windus,

Bira, L., Evans, T. M. and Vanderford, N.L. (2019). Mental health in academia: an invisible crisis. Physiology News Magazine, 2019(115), retrieved from

https://www.physoc.org/magazine-articles/mental-health-in-academia-an-invisible-crisis/.

Bodnar, Adam, Corbett, Richard, and Nekrasovski, Dmitry. 2004. AROMA: Ambient Awareness through Olfaction in a Messaging Application. ICMI04, Pennsylvania, USA. 12-15, October, 2004.

Bolton, Ruth., Gustafsson, Aders., McColl-Kennedy, Janet, Sirianni, Nancy, and Tse, David. 2014. "Small Details that Make Big Differences" Journal of Service Management, 25, no.2: 253-274.

Clawson, J., Pater, J.A. Miller, A.D., Maynatt, E.D. and Mamykina, L. (2015). No longer wearing: investigating the abandonment of personal health-tracking technologies on Craigslist. UBICOM, Osaka, Japan

Desmet, Pieter and Hekkert, Paul. 2007. "Framework of Product Experience. International Journal of Design, 1, no.1:1-10.

Frayling, Christopher. 1993 "Research in Art and Design. Royal College of Art Research Papers, 1, no.1:1-5.

Gartner. "Gartner Survey Shows Wearable Devices Need to be More Useful." Accessed 3r March, 2019. http://www.gartner.com/newsroom/id/3537117 on 03.03.2019.

Koskinen, Ilpo., Zimmerman, John., Binder, Thomas., Redstrom, Johan. and Wensveen, S.tephan Design research through practice: from the lab, field, and showroom. MA, USA: Elsevier, 2011.

Landrum, S. (2017). Why Millennials are struggling with mental health at work. Forbes, Retrieved from https://www.forbes.com/sites/sarahlandrum/2017/01/17/why-millennialsare-struggling-with-mental-health-at-work/\#4fc6d57b4a9b on 20.09.2019.

Lazar, A., Koehler, C., Tanenbaum, J. and Nguyen, D.H. (2015). Why we use and abandon smart devices. UBICOMP, Osaka, Japan

Lenz, Eva., Diefenbach, Sarah. and Hassenzahl, Marc. 2014. Aesthetics of Interaction - a Literature Synthesis. NORDiCHI14, Helsinki, Finland, 28-30 October, 2014.

Leonardi, Paul.M. 2011. When Flexible Routines Meet Flexible Technologies: Affordance, Constraint, and the Imbrication of Human and Material Agencies. MIS Quarterly, 35, no.1: 147-167.

McColl-Kennedy, J.R., Danaher, T.S., Gallan, A.S., Orsignher, C., Lervik-Olsen, L. and Verma, R. (2017). How do you feel today? Managing patient emotions during healthcare experiences to enhance well-being. Journal of Business Research, 79(2017), $247-259$. 
Russell, James. A. 1980. A Circumplex Model of Affect. Journal of Personality and Social Psychology, 39, no.6: 1161-1178.

Sheth, Jagdish. N., Newman, Bruce. I., and Gross, Barbara. L. 1991. Why We Buy What We Buy: A Theory of Consumption Values. Journal of Business Research, 22, no.2: 159-170.

Xiang, Wei, Chen, Shi, Sun, Lingyun, Cheng, Shiwei, and Bov. Jr. Machael. 2016. Odor Emoticon: An Olfactory Application that Conveys Emotions. International Journal of HumanComputer Studies, 91, no.2016: 52-61.

Zomerdijk, Leonieke. G. and Voss, Christopher. A. (2010). Service Design for ExperienceCentric Services. Journal of Service Research, 13, no.1: 67-82.

Bio

Cecilia is a PhD candidate in Service Design at the Royal College of Art in London, the UK.

Contact Details

cecilia.lee@network.rca.ac.uk 